This article has not yet been peer reviewed. It is likely to change prior to final publication in a peer-reviewed journal. Any citation and interpretation of results herein should be made with this consideration

\title{
Reward learning and statistical learning independently influence attentional priority of salient distractors in visual search
}

\author{
Mike E. Le Pelley ${ }^{1 *}$, Rhonda Ung ${ }^{1 *}$, Chisato Mine ${ }^{1,2,3}$, Steven B. Most ${ }^{1}$, \\ Poppy Watson ${ }^{1}$, \& Jan Theeuwes ${ }^{4}$
}

1. School of Psychology, UNSW Sydney, Australia

2. Human-Centered Mobility Research Center, Department of Information Technology and Human Factors, National Institute of Advanced Industrial Science and Technology (AIST), Tsukuba, Japan

3. Japan Society for the Promotion of Science

4. Department of Experimental and Applied Psychology, Vrije Universiteit, Amsterdam, The Netherlands.

* These authors contributed equally to this work

\section{Corresponding author:}

A/Prof Mike Le Pelley

School of Psychology, UNSW Sydney

Sydney NSW 2052

Australia

\section{Conflict of interest:}

The authors declare no conflicts of interest.

\section{Funding:}

This research was funded by Australian Research Council grant DP200101314. Poppy Watson was supported by an Australian Research Council Discovery Early Career Researcher Award (DE200100591). 


\begin{abstract}
Existing research demonstrates different ways in which attentional prioritisation of visual stimuli is shaped by prior experience: reward learning renders signals of high-value outcomes more likely to capture attention than signals of low-value outcomes, whereas statistical learning can produce attentional suppression of the location in which salient distractor items are likely to appear. The current study combined manipulations of the value and location associated with distractors in visual search to investigate whether these different effects of selection history operate independently, or interact to determine overall attentional prioritisation of salient distractors. In Experiment 1, high- and low-value distractors most frequently appeared in the same location; in Experiment 2, high- and lowvalue distractors typically appeared in distinct locations. In both experiments, effects of distractor value and location were additive, suggesting that attention-promoting effects of value and attention-suppressing effects of physical salience independently modulate overall attentional priority. Our findings are consistent with a view that sees attention as mediated by a common priority map that receives and integrates separate signals relating to physical salience and value.
\end{abstract}

Keywords: attention; reward; suppression; statistical learning; visual search 


\section{Introduction}

As we interact with the world, we are confronted with an continuous stream of incoming sensory information. Attention refers to the set of cognitive mechanisms that act to prioritise certain aspects of this sensory input for further analysis and action, and to suppress other aspects that might otherwise interfere with our ongoing information processing. $\mathrm{A}$ traditional view sees attentional selection as reflecting the balance between goal-directed processes (based on our intentions, and task-specific demands) and stimulus-driven processes (wherein highly distinctive and salient stimuli capture our attention automatically, regardless of our current goals: Itti \& Koch, 2001; Yantis, 2000). However, more recent work has extended this view by demonstrating that our attention is shaped at a fundamental level by our previous experiences with stimuli (for reviews, see Awh et al., 2012; Failing \& Theeuwes, 2018; Le Pelley et al., 2016).

One example of this influence of prior experience on attention is seen in a recent body of work demonstrating that the likelihood that a stimulus will receive attentional priority is modulated by previous learning about the relationship between that stimulus and events of motivational significance: rewards and punishments (for reviews, see Anderson, 2016; Le Pelley et al., 2016; Rusz et al., 2020; Watson, Pearson, Wiers, et al., 2019). This effect has been demonstrated across a range of procedures; here we focus on a procedure developed by Le Pelley, Pearson, Griffiths and Beesley (2015; see also Watson, Pearson, Most, et al., 2019), which was a variant of the additional singleton task (Theeuwes, 1992). On each trial participants searched for and responded to a diamond target among circles. Correct responses earned reward, and the faster the response, the larger the reward; errors resulted in a loss of the amount that would otherwise have been won. On the majority of trials, one of the circles in the search display was coloured either blue or orange; all other shapes were 
grey. We refer to the colour-singleton circle as the distractor to distinguish it from the other (grey) circles in the display. If the distractor circle appeared in the high-value colour (say blue) this signalled that the current trial was a bonus trial on which reward/loss would be multiplied by a factor of 10; if the distractor was in the low-value colour (orange in this case) the current trial was not a bonus trial. Notably, while the distractor signalled the magnitude of the available reward, it was not the target that participants responded to in order to receive that reward. The optimal strategy in this task is to ignore the distractors and respond to the target as rapidly as possible, since this would yield highest earnings. Nevertheless, responses to the target were significantly slower (but not more accurate) for trials featuring a high-value distractor than for trials with a low-value distractor. This suggests that the highvalue distractor was more likely to receive attentional priority, interfering with search for the target - even though this behaviour was counterproductive, as it meant participants earned less on high-value trials than would otherwise have been the case. In other words, these findings suggest that learning about the value associated with a stimulus can change the likelihood that it captures attention in the future (Anderson et al., 2011; Le Pelley et al., 2015; Pearson et al., 2015; Pearson et al., 2016; Watson, Pearson, Chow, et al., 2019), a phenomenon termed value-modulated attentional capture (VMAC).

Findings such as these have led to the suggestion that effects of value feed into a common attentional priority map (Awh et al., 2012; Belopolsky, 2015; Failing \& Theeuwes, 2018; Pearson et al., 2016; Theeuwes, 2018). This map is thought to integrate information based on the physical salience of stimuli (the distinctiveness of their physical features: colour, luminance, shape etc), goal-directed selection biases (for task-relevant information), and effects of prior experience (such as association with reward or punishment), to determine the overall attentional priority of each location in a scene. 
The effect of value on attention (as demonstrated in the VMAC effect) illustrates a case in which prior experience can increase the likelihood that certain stimuli will capture attention. Recent research suggests that experience can also reduce the likelihood that stimuli will capture attention in future. This research demonstrates that statistical learning about the likely location in which a salient distractor stimulus will appear can induce location-specific suppression, such that items appearing in that location will be less likely to receive attention than items appearing in less-expected locations (Failing, FeldmannWüstefeld, et al., 2019; Wang et al., 2019; Wang \& Theeuwes, 2018a, 2018b). For example, Wang and Theeuwes (2018a) used an additional-singleton task in which a colour-singleton distractor appeared most frequently in one particular location, and less frequently in other locations. Participants were faster to detect the (shape-defined) target when the search display contained a distractor in the 'frequent' location, than when the distractor was in one of the 'rare' locations. This finding was taken to suggest that statistical learning about the most probable distractor location created suppression at that location on the attentional priority map, such that items appearing there competed less for attentional priority than did items at other locations. Consistent with this idea of items at the frequent-distractor location being suppressed, Wang and Theeuwes (2018a) also found that on occasional trials without a colour-singleton distractor, responses to the target were slower when that target appeared in the location in which a distractor had frequently appeared, than when the target appeared in one of the other, 'rare' locations. Further research has shown that the effect reflects learning about the likely location of distractors (again in line with the idea of learned suppression of distractors), rather than the likely location of the target (Failing, Wang, et al., 2019), and is independent of top-down attentional control (Gao \& Theeuwes, 2020). 
The studies noted in the previous paragraph have demonstrated that statistical learning can generate suppression of physically salient distractors (i.e., distractors that are distinctive based on their physical features; colour in these studies). This raises the question of whether the suppression that is generated by statistical learning is specific to physical salience, or whether it applies equally to other aspects that might render a stimulus salient namely its association with valued events. We noted earlier a view that sees attention as mediated by a common priority map that receives and integrates signals relating to physical salience and selection history (which includes effects of value). On this account, suppression applied to a particular location on the common priority map should reduce attention to any type of stimulus presented at that location, regardless of whether its salience derives from its physical features or its association with reward. That is, this account anticipates that effects of value on attentional capture should be independent of suppression generated through statistical learning about the likely locations in which distractors will appear. The current study aimed to test this idea.

\section{Experiment 1}

Experiment 1 effectively combined the statistical-learning procedure of Wang and Theeuwes (2018a) with the reward-learning procedure of Watson, Pearson, Most, et al. (2019). Participants completed an additional-singleton task in which they searched for and responded to a shape-singleton target. The colour of a singleton distractor in the search display signalled whether a high or low reward was available. Regardless of its colour, this distractor was more likely to appear in one of the stimulus locations than others. We anticipated that-consistent with prior research—-the high-value distractor would become more likely to capture attention (and hence slow responding to the target) than the lowvalue distractor, and that distraction would be reduced when the distractor appeared in its 
typical location versus an atypical location (suggesting suppression of the typical location). The key question was whether these two effects would influence responding independently, or whether they would interact.

\section{Method}

\section{Participants and Apparatus}

Previous studies have found medium to very large effect sizes $\left(d_{z}=0.54-2.20\right)$ for the influence of reward on attention in tasks similar to that used here (e.g., Le Pelley et al., 2015; Pearson et al., 2015, 2016; Watson et al., 2019a, 2019b, 2020), and large effect sizes $\left(d_{z}=0.69-2.15\right)$ for the influence of statistical learning about distractor location (Failing, Feldmann-Wüstefeld, et al., 2019; Failing \& Theeuwes, 2020; Failing, Wang, et al., 2019). Consequently we aimed to recruit at least 29 participants; G*Power (with default settings for correlation among repeated measures and nonsphericity correction) revealed that this would give power of .80 to detect medium-sized within-subjects effects $\left(d_{z}=0.54\right)$. A total of 32 UNSW Sydney students (20 females, 11 males, 1 other; age $M=19.6$ years, SEM $=0.3$ ) completed the task for course credit; they were also informed that the top-scoring half of participants (see below) would receive a AU\$20 supermarket voucher. The task was run online in a web browser, with stimulus presentation controlled by jsPsych (de Leeuw, 2015), which provides accurate response-time measurement (de Leeuw \& Motz, 2016). Participants completed the experiment using personal computers; the task was not compatible with phones or tablets. All research reported here was approved by the UNSW Sydney Human Research Ethics Advisory Panel (Psychology); experiment code and raw data are available via the Open Science Framework at https://osf.io/zg3nr/. 


\section{Stimuli and Design}

All stimuli were presented on a black background. Each trial (see Figure 1) began with a central fixation cross. After 400 ms, the search display appeared. This display contained eight shapes $(72 \times 72$ pixels): either one diamond and seven circles, or one circle and seven diamonds (randomly determined on each trial), arranged evenly around screen centre at an eccentricity of 140 pixels. Each shape contained a grey (RGB: $[70,70,70])$ line segment oriented horizontally or vertically (randomly determined). On most trials, one of the nonsingleton shapes was coloured either blue (RGB: $[37,141,165])$ or orange (RGB: $[193,95,30])$; all other shapes were grey (RGB: $[70,70,70])$. We term the coloured shape the distractor. Assignment of blue and orange to the role of high-value and low-value colours was randomly determined for each participant. 


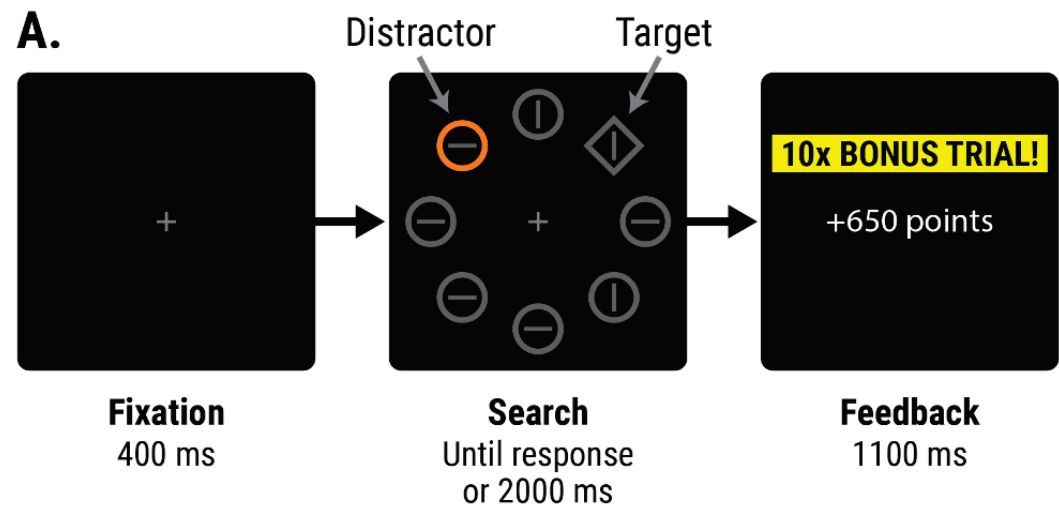

\section{B. Experiment 1}

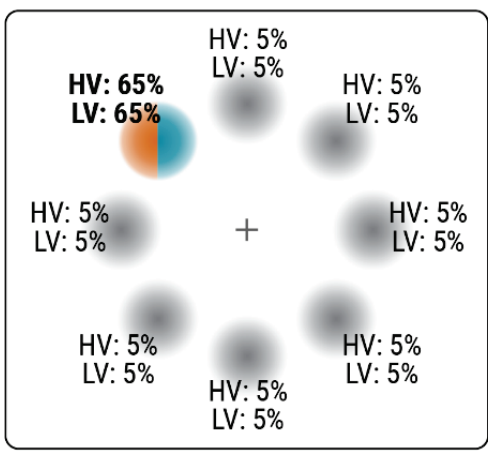

Frequent location high-value distractor
C. Experiment 2

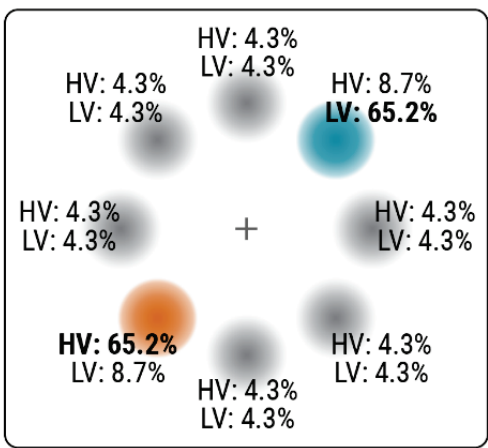

Frequent location low-value distractor
Rare location

Figure 1. (A) Example trial of the search task. Participants' task was to respond according to the orientation of the line in the target shape, which was a shape singleton: either a diamond among circles (as illustrated here) or a circle among diamonds. The display could contain a colour-singleton distractor shape, which was coloured orange or blue. Lower panels show a schematic representation of the manipulation of distractor location in (B) Experiment 1 and (C) Experiment 2. The frequent location of the high-value (HV) and low-value (LV) distractors are shown in orange and blue, respectively; rare locations are shown in grey. Percentages at each location give the probability that each distractor type would occur at that location (when that distractor type was present in the search display). In Experiment 1, both high- and low-value distractors appeared most frequently at the same location; in Experiment 2, the high- and low-value distractors appeared most frequently at different locations (which were opposite one another in the search display). Note that the frequent distractor locations shown here are an example: these locations were chosen randomly for each participant.

Participants' task was to report the orientation of the line within the shape-singleton target as quickly as possible—by pressing either ' $\mathrm{C}$ ' (horizontal) or ' $\mathrm{M}$ ' (vertical) — with faster correct responses earning more points. For trials with a distractor in the low-value colour, and for trials with no colour-singleton distractor in the search display (distractor-absent 
trials), correct responses earned 0.1 points for every ms that response time (RT) was below 1400 ms (so an RT of 600 ms would earn 80 points). Trials in which the display contained a high-value distractor were 'bonus trials', and points were multiplied by 10 (so an RT of 600 ms would earn 800 points). Correct responses with RT greater than 1400 ms earned no points, and errors resulted in loss of the points that otherwise would have been won. Responses with RT less than 150 ms were considered to be anticipations. The search display remained on-screen until a response was made or the trial timed-out (after $2000 \mathrm{~ms}$ ). A feedback screen then appeared. If an anticipation had been made, feedback stated "Please do not anticipate which response to make" and appeared for $2500 \mathrm{~ms}$. In all other cases, feedback appeared for $1100 \mathrm{~ms}$. If the trial had timed-out, feedback stated "Too slow. 0 points". Otherwise, if the response was correct, feedback showed the number of points won (e.g., "+80 points"); if the response was incorrect, feedback showed "ERROR" and the number of points lost (e.g., "ERROR: LOSE 350 points"). On trials with a high-value distractor, feedback was accompanied by a box labelled "10x bonus trial!". Following feedback, the next trial began after a blank inter-trial interval of $1000 \mathrm{~ms}$.

When the search display contained a colour-singleton distractor-regardless of its colour - this distractor was more likely to appear in one of the display locations (termed the frequent location) than the others (rare locations). The frequent location remained the same throughout the task, and was chosen randomly for each participant.

Each block of the task contained 56 trials: 20 trials with a high-value distractor, 20 trials with a low-value distractor, and 16 distractor-absent trials. Of the 20 trials in each block with the high-value distractor, the distractor appeared 13 times in the frequent location, and once in each of the other seven (rare) locations. The same applied for trials with the low-value distractor. Hence of the trials featuring a distractor, $65 \%$ had this distractor in the frequent 
location and $35 \%$ had the distractor in one of the rare locations. Trial order within each block was random, as was the location of the target on each trial.

\section{Procedure}

Participants were informed that their aim was to earn as many points as possible, and that the top-scoring half of participants would win a AU\$20 supermarket voucher. As an additional motivation, participants were told that points would unlock 'medals'. For every 24,000 points, participants unlocked a new medal (in the order bronze, silver, gold, platinum, diamond, and elite). Based on mean RTs from pilot work, this would mean that the bestperforming $\sim 10 \%$ of participants would unlock the 'elite' medal.

Instructions prior to the search task informed participants (1) that faster (correct) responses would earn more points, (2) that when a circle in the high-reward colour appeared in the search display it would be a bonus trial on which points were multiplied by 10 , and (3) that when a circle in the low-reward colour appeared it would not be bonus trial. Checkquestions verified that participants understood these instructions: participants were required to respond correctly to these questions before they could continue. No mention was made of the fact that distractors would appear more commonly in one of the locations. Participants then completed 16 blocks (896 trials), taking a break after each block during which they were shown their total number of points, and an animation presented any medals unlocked since the previous break.

Previous studies of statistical learning in visual search have examined whether, following the search task, participants were explicitly aware that the distractor appeared more frequently in one particular location; data relating to (a lack of) awareness have been used to argue that learning of statistical regularities is implicit (e.g., Failing, FeldmannWüstefeld, et al., 2019; Wang et al., 2019; Wang \& Theeuwes, 2018a). We remain wary of 
drawing such conclusions (see General Discussion); nevertheless, for consistency with previous research we probed participants' explicit knowledge following the current search task. First, participants were asked whether they thought the coloured shape had been equally likely to appear in each of the eight stimulus locations, or if it had been more likely to appear in some location(s) than others. They were then asked to rate their confidence in this choice on a scale from 1 (least confident) to 5 (most confident). After making their response, participants were informed that the coloured shape had actually been more likely to appear in one of the eight locations than the others. They were then asked to select (1) whether this frequent location had been in one of the three upper locations in the search display, one of the two middle locations, or one of the three bottom locations; (2) whether the frequent location had been in one of the three left-hand locations, the two central locations, or the three right-hand locations; and (3) in which specific location the distractor had been most likely to appear.

\section{Results}

For two participants, more than a third of trials in the search task had invalid responses (anticipations or time-outs); all data from these participants were excluded from further analyses. After removal of invalid responses, two participants had a mean accuracy below $60 \%$; these poorly performing participants were also excluded. For remaining participants ( $n=28$ ), following our previous protocols (Le Pelley et al., 2015; Watson, Pearson, Most, et al., 2019) we discarded data from the first two trials after each break, time-outs (2.6\% of all trials), and anticipations ( $0.2 \%$ of all trials); after exclusions, mean accuracy for these participants was $82.6 \%(S E M=1.5 \%)$. Analysis of RTs used correct responses only. 


\section{Distractor-present trials}

Figure 2 shows mean RT and error rate for search trials featuring a colour-singleton distractor. Analysis of the RT data via $2 \times 2$ ANOVA with factors of distractor value (high-value vs. low-value) and distractor location (frequent vs. rare) revealed a main effect of distractor value, $F(1,27)=34.9, p<.001, \eta_{p}^{2}=.564$, with slower responses for high-value than lowvalue trials, indicating an attentional bias towards the high-value distractor. There was also a main effect of distractor location, $F(1,27)=88.9, p<.001, \eta_{p}^{2}=.767$, with faster responses when the distractor appeared in the frequent location than when it appeared in one of the rare locations, indicating that the salient distractor was less likely to capture attention when it occurred in the frequent location. Notably, the interaction of value and location was not significant, $F(1,27)=0.04, p=.848, \eta_{p}^{2}=.001$. To assess the strength of support for the null hypothesis, we reanalysed these data using Bayesian ANOVA, conducted in jamovi (The jamovi project, 2020) with the default prior. We assessed evidence for the interaction by comparing Bayes Factors for the model including both main effects and interaction, versus the model with main effects only. This revealed a Bayes Factor for inclusion of the interaction of $B F_{10}=0.204$, indicating substantial support for the null hypothesis (i.e., that effects and value and location are independent) over the alternative hypothesis (Jeffreys, 1961). 


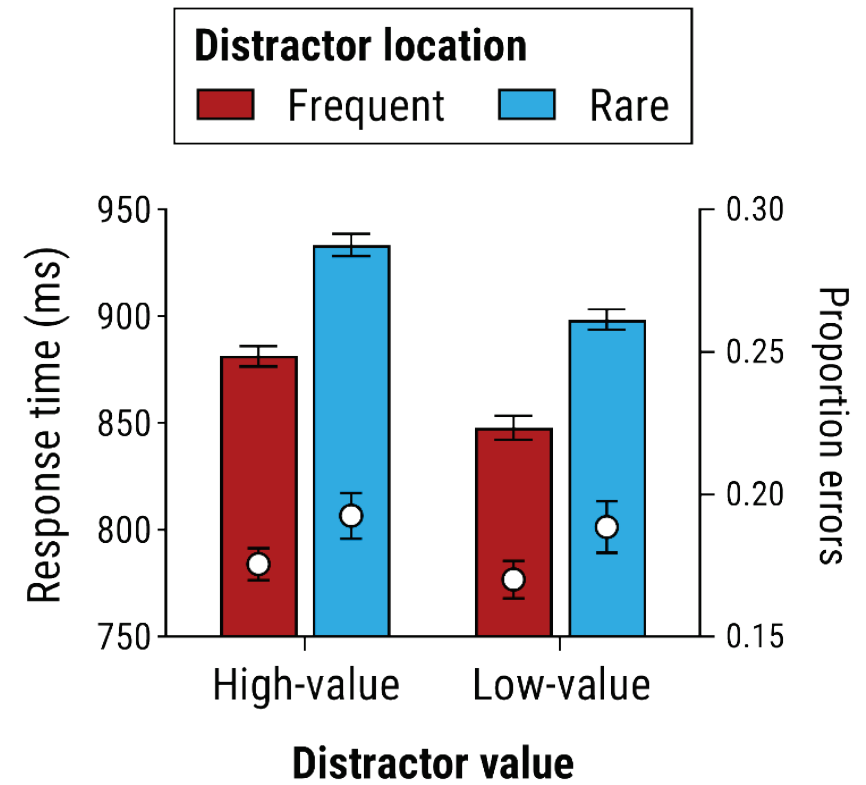

Figure 2. Mean response times and proportion of errors for trials of the search task featuring a colour-singleton distractor in Experiment 1, as function of the value of the reward signalled by the distractor, and the location of that distractor. In this and all other figures, bars show mean response time, superimposed white circles show mean proportion of errors, and error bars show within-subjects standard error of the mean (Morey, 2008).

Analysis of error rates using similar ANOVA revealed a nonsignificant main effect of value, $F(1,27)=0.31, p=.581, \eta_{p}^{2}=.011$. The main effect of location was significant, $F(1,27)=5.95, p=.022, \eta_{p}^{2}=.180$, with fewer errors when the distractor appeared in the frequent location than a rare location. Again the value $\times$ location interaction was nonsignificant, $F(1,27)=0.01, p=.907, \eta_{p}^{2}<.001$, with Bayesian analysis revealing substantial support for the null hypothesis, $B F_{10}=0.249$.

\section{Distractor-absent trials}

To investigate further the suppression of the frequent location generated by statistical learning, following Wang and Theeuwes (2018a) we analysed performance on distractorabsent trials as a function of whether the target was located at the frequent location or one of the rare locations; data are shown in Figure 3. Responses were significantly slower, 
$t(27)=2.79, p=.010, d_{z}=0.527$, and less accurate, $t(27)=2.35, p=.026, d_{z}=0.444$, when the target appeared in the frequent distractor location than in the average of the rare locations.

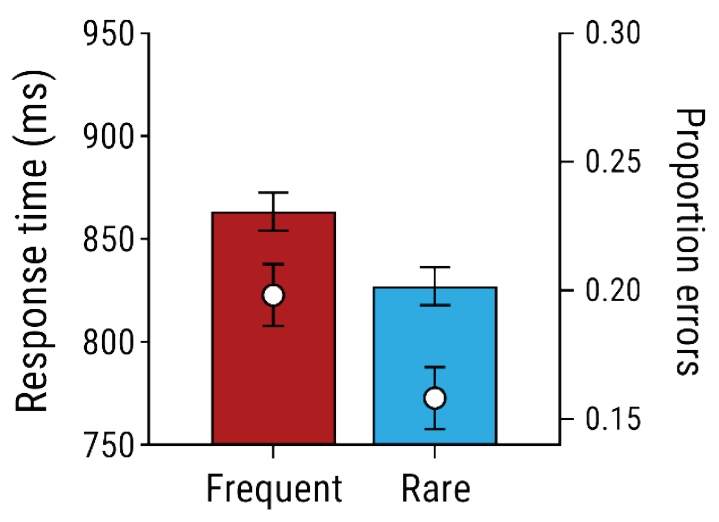

Target location

Figure 3. Mean response times and proportion of errors for distractor-absent trials (i.e., trials in which the search display did not contain a colour-singleton distractor) of Experiment 1 , as a function of whether the target appeared in the location in which the salient distractor had typically appeared (frequent) versus the average of the locations in which the distractor seldom appeared (rare).

\section{Distractor-present versus distractor-absent trials}

Mean response time across all distractor-absent trials was $832 \mathrm{~ms}(S E M=24 \mathrm{~ms})$. A set of Bonferroni-corrected, pairwise t-tests revealed that response time in each of the distractor-present conditions (see Figure 2) was significantly slower than on distractor-absent trials, all $t(27)>3.21, p<.014, d_{z}>0.60$. Thus the presence of any colour-singleton distractor, regardless of its value or location, impaired performance to some degree, indicating that suppression of distractors was not complete in any condition.

\section{Awareness of statistical regularities}

As noted earlier, we have reservations about the way in which participants' (lack of) awareness of statistical regularities has been interpreted as evidence for implicit learning, 
and the issue of awareness was not a focus of the current research. Nevertheless, we report data from the awareness tests for the sake of completeness. Of the 28 participants, 18 reported that they thought the distractor had appeared more often in some location(s) than others (versus being random); for these 18 participants, mean confidence was relatively low, at $2.56(S E M=0.22)$ on the 5-point scale. After being told that the distractor was in fact more likely to occur in one location, 12 of the 28 participants correctly selected the frequent location when asked to do so; given eight possible locations, this was significantly above chance by a binomial test, $p<.001$. However, repeating analysis of search RTs while excluding the 12 participants who correctly chose the frequent location left the pattern of significant and nonsignificant results unchanged.

\section{Discussion}

Consistent with previous findings (Albertella, Le Pelley, et al., 2019; Albertella, Watson, et al., 2019; Le Pelley et al., 2015; Watson, Pearson, Most, et al., 2019), responding to the target in Experiment 1 was significantly slower (but no more accurate) when the search display contained a high-value distractor than when it contained a low-value distractor. This implies that participants were more likely to attend to the high-value distractor, thus interfering with search for the target. This influence of distractor value on attention (and performance) was counterproductive, since response times influenced the points earned in the search task - by responding more slowly (but no more accurately) on trials in which the highest rewards were available, participants lost out disproportionately.

Moreover, search performance was significantly better (faster and more accurate) when the colour-singleton distractor appeared in the frequent location than in a rare location. This finding is again consistent with prior research (e.g., Failing, Feldmann-Wüstefeld, et al., 2019; Wang et al., 2019; Wang \& Theeuwes, 2018a, 2018b), and has been suggested to reflect the 
development of attentional suppression at the frequent location, such that salient items presented at this location compete less for attentional priority than items at other locations. In line with this idea (and again as in prior research), we also found that on distractor-absent trials, participants were slower to respond to the target when it appeared in the location in which a salient distractor had frequently appeared versus a location in which a salient distractor had rarely appeared.

Importantly, the effects on performance of distractor value and distractor location were additive: we found no evidence of an interaction between the effects of these factors, and Bayesian analyses provided support for the null hypothesis. The implication is that reward learning and statistical learning exert independent effects on attentional prioritisation, consistent with the idea that these processes have separate inputs to a common attentional priority map. Our results indicate that the amount of suppression applied to the frequent location on a given trial was not modulated by the salience of the item appearing at that location: high-value distractors were more salient than low-value distractors (they interfered more with search for the target), and yet they did not elicit greater suppression. This finding is in line with the idea of suppression being proactive: on this account, a fixed, 'negative priority' input is applied at the frequent location prior to appearance of the search display, and subtracts from the salience of whatever item appears at that location). By contrast, our findings are less consistent with the idea that suppression is driven by reaction to the salience of a presented distractor - or at least suggests that any such reactive suppression is subject to a low ceiling such that presentation of a high-value distractor swamps the limited suppression that can be applied. 


\section{Experiment 2}

In Experiment 1 we examined the case in which there was a single location in which both types of distractor-high-value and low-value-were most likely to appear. Participants did not know in advance which type of distractor would appear on a given trial, and under these conditions it makes sense that the attentional system might develop a certain, fixed level of suppression of the frequent location that would apply equally regardless of the value (and colour) of the distractor presented at that location. In Experiment 2 we instead used a search task in which high-value distractors were likely to appear in one location (termed the frequentHigh location), while low-value distractors were likely to appear in a second location (frequentLow), in order to investigate whether this difference in experience would lead to different levels of suppression at the frequentHigh and frequentLow locations. Under these conditions there should be a greater drive to develop suppression at the frequentHigh location, since this location will typically hold very salient high-value distractors, and suppressing attention to these distractors is particularly beneficial because it would result in larger rewards under the task's payoff structure. By contrast, if effects of reward learning and statistical learning on attention are independent (as suggested by the results of Experiment 1), then we should expect to see equal evidence of suppression at both frequentHigh and frequentLow locations.

\section{Method}

\section{Participants and Apparatus}

A total of 44 online participants ( 14 females, 28 males, 2 other; age $M=27.1$ years, $S E M=1.2$ ) were recruited via Prolific (www.prolific.co). Participants received $£ 7.50$ for 
completing the task, and were informed that the top-scoring $25 \%$ of participants would receive a bonus of $£ 4$. Other aspects were as for Experiment 1 .

\section{Stimuli, Design and Procedure}

Stimuli, design and procedure were as for Experiment 1, with exceptions as outlined here. For each participant one of the eight stimulus locations was randomly chosen to serve as the frequentHigh location; the frequentLow location was in the diametrically opposite position (cf. Failing, Feldmann-Wüstefeld, et al., 2019).

Each block of the task contained 56 trials: 23 trials with the high-value distractor, 23 trials with the low-value distractor, and 10 distractor-absent trials. Of the 23 trials in each block with the high-value distractor, it appeared 15 times in the frequentHigh location, twice in the frequentLow location, and once in each of the other six (rare) locations. Likewise, of the 23 trials in each block with the low-value distractor, it appeared 15 times in the frequentLow location, twice in the frequentHigh location, and once in each of the other six (rare) locations. Consequently each distractor appeared $65.2 \%$ of the time in its own most frequent location, $8.7 \%$ of the time in the most frequent location of the other distractor, and $4.3 \%$ of the time in each of the other six locations. Target location was chosen randomly on distractor-present trials, but was constrained for distractor-absent trials: of the 10 distractorabsent trials in each block, the target appeared twice in each of the frequentHigh and frequentLow locations, and once in each of the other six locations. Participants completed 16 blocks of trials (896 trials total).

Following the search task, as in Experiment 1 participants were asked whether they thought the coloured shape had been equally likely to appear in each of the eight stimulus locations, or if it had been more likely to appear in some location(s) than others, and rated their confidence in this choice. Participants were then informed that the blue shape had been 
most likely to appear in one location, and the orange shape in a different location. They were then asked to indicate in which location each type of distractor (high-value and low-value colours) had been most likely to appear, in random order.

\section{Results}

No participants had more than a third of trials in the search task with invalid responses (anticipations or time-outs). After removal of invalid responses, three participants had a mean response accuracy below $60 \%$; these poorly performing participants were excluded from subsequent analyses. For remaining participants $(n=41)$, as in Experiment 1 we discarded data from the first two trials after each break, time-outs ( $0.79 \%$ of all trials), and anticipations ( $0.04 \%$ of all trials); after exclusions, mean accuracy for included participants was $86.4 \%(S E M=0.8 \%)$.

\section{Distractor-present trials}

Figure 4 shows mean RT (for correct responses) and error rate for search trials featuring a colour-singleton distractor. Data are grouped according to whether the distractor appeared in its own most frequent location (i.e., high-value distractor in frequentHigh location; low-value distractor in frequentLow location: labelled the match condition), or in the most frequent location of the other type of distractor (high-value distractor in frequentLow location and vice versa: mismatch condition), or in one of the rare locations. For this analysis we excluded any trials in which the target appeared at either the frequentHigh or frequentLow location, i.e., for all trials in this analysis, the target appeared at a 'rare' location. 

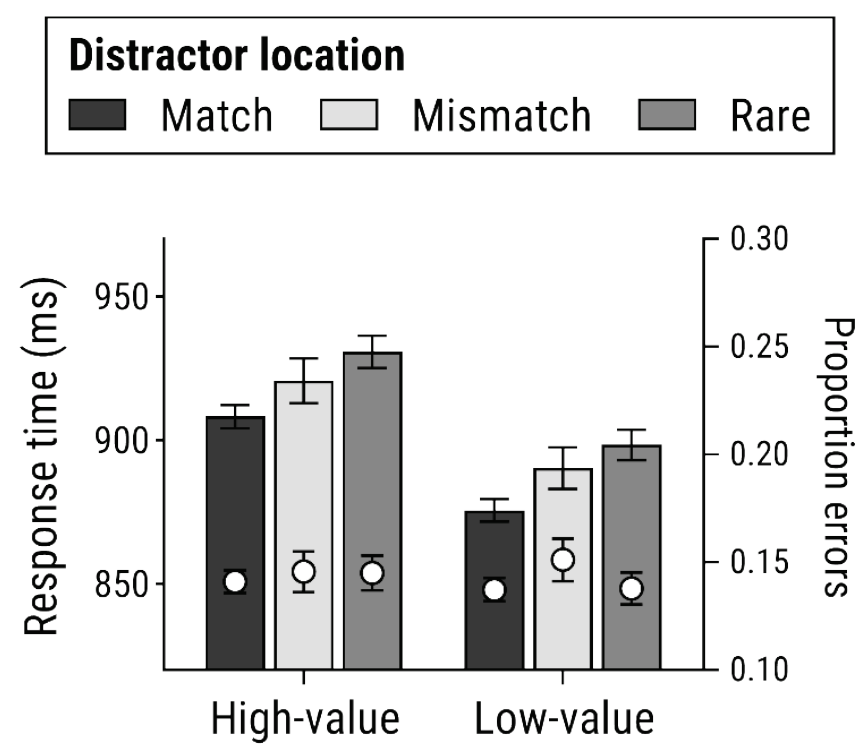

Distractor value

Figure 4. Mean response times and proportion of errors for trials of the search task featuring a colour-singleton distractor in Experiment 2, as a function of the value of the reward signalled by the distractor, and the location of that distractor: whether the distractor appeared in its own most frequent location (i.e., high-value distractor in frequentHigh location; low-value distractor in frequentLow location: labelled match), or in the most frequent location of the other type of distractor (high-value distractor in frequentLow location and vice versa: mismatch), or in one of the rare locations.

RT data were analysed via $2 \times 3$ ANOVA with factors of distractor value (high-value vs. low-value) and distractor location (match, mismatch, rare). This revealed a main effect of distractor value, $F(1,40)=27.0, p<.001, \eta_{p}{ }^{2}=.403$, with slower responses for high-value than low-value trials, indicating a general value-related attentional bias. There was also a main effect of distractor location, $F(2,80)=8.88, p<.001, \eta_{p}{ }^{2}=.182$, with Tukey HSD tests revealing that responses were significantly faster in the match condition than the mismatch condition, $t(80)=2.50, p=.038$, and in the match condition than the rare condition, $t(80)=4.19, p<.001$; there was no significant difference in response time in the mismatch and rare conditions, $t(80)=1.69, p=.217$. Returning to the ANOVA, there was no significant value $\times$ location interaction, $F(2,80)=0.03, p=.973, \eta_{p}^{2}<.001 ;$ that is, the pattern of 
suppression across match, mismatch and rare locations did not differ significantly depending on whether a high- or low-value distractor appeared. As in Experiment 1, we assessed the strength of evidence for the null interaction using Bayesian ANOVA, by comparing Bayes Factors for the model including both main effects and interaction, versus the model with main effects only. This revealed a Bayes Factor for inclusion of the interaction of $B F_{10}=0.077$, indicating strong evidence for the null hypothesis (that effects of value and location are independent) over the alternative hypothesis (Jeffreys, 1961).

Analysis of error rates using similar ANOVA revealed a no significant mains or interaction, all $F s<1$, indicating that differences in RTs were likely not a consequence of speed-accuracy trade-offs.

\section{Distractor-absent trials}

To investigate further the suppression of the frequent distractor locations generated by statistical learning, we used one-way ANOVA to analyse performance on distractor-absent trials as a function of whether the target was positioned at the frequentHigh location, the frequentLow, or one of the rare locations (see Figure 5). This revealed a significant effect of target location, $F(2,80)=3.44, p=.037, \eta_{p}^{2}=.079$. Planned pairwise t-tests revealed that responses were significantly faster when the target appeared in a rare location than when it appeared in the frequentHigh location, $t(40)=3.12, p=.003, d_{z}=0.487$, or the frequentLow location, $t(40)=2.07, p=.045, d_{z}=0.322$. However, there was no significant difference in RT between trials with the target in the frequentHigh versus frequentLow location, $t(40)=0.66$, $p=.513, d_{z}=0.103$, with a Bayesian t-test revealing substantial support for the null hypothesis, $B F_{01}=0.207$. ANOVA analysis of corresponding data for proportion of errors revealed no significant effect of target location, $F(2,80)=0.06, p=.946, \eta_{p}{ }^{2}=.001$. 


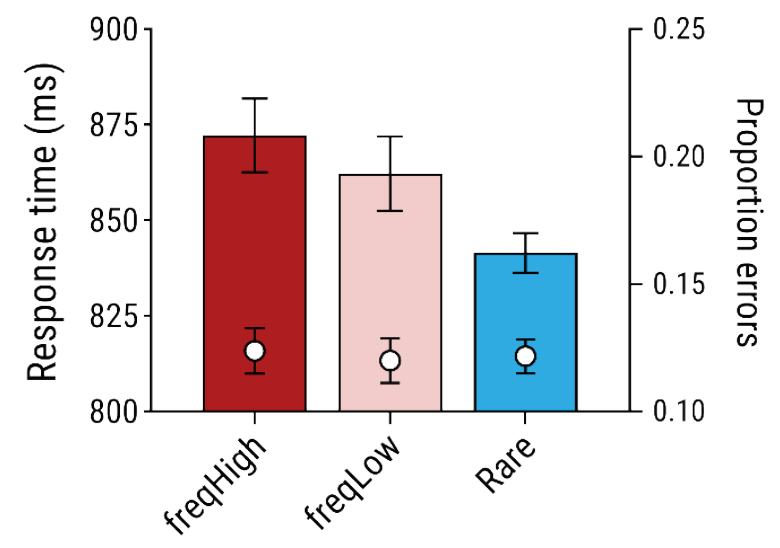

Target location

Figure 5. Mean response times and proportion of errors for distractor-absent trials (i.e., trials in which the search display did not contain a colour-singleton distractor) of Experiment 1 , as a function of whether the target appeared in the location in which the high-value distractor had typically appeared (freqHigh), or the location in which the low-value distractor had typically appeared (freqLow), versus the average of the remaining six locations in which the distractors seldom appeared (rare).

\section{Distractor-present versus distractor-absent trials}

Mean response time across all distractor-absent trials was $851 \mathrm{~ms}(S E M=18 \mathrm{~ms})$. A set of Bonferroni-corrected, pairwise t-tests revealed that response time in each of the distractor-present conditions (see Figure 4) was significantly slower than on distractor-absent trials, all $t(40)>5.68, p<.001, d_{z}>0.88$. Thus the presence of any colour-singleton distractor, regardless of its value or location, impaired performance to some degree, indicating that suppression of distractors was not complete in any condition.

\section{Awareness of statistical regularities}

Of the 41 participants, 16 reported that they thought the distractors had appeared more often in some location(s) than others (versus being random); for these 18 participants, mean confidence was moderate, at $3.38(S E M=0.18)$ on the 5-point scale. After being told that each distractor colour was in fact most likely to occur in a distinct location, 21 of the 41 
participants correctly identified the frequentHigh location for the high-value distractor, and 18 participants correctly identified the frequentLow location for the low-value distractor. Binomial tests revealed that performance for both types of distractor was significantly above chance, $p s<.001$, and a McNemar test indicated that selection of the correct location for the high- versus low-value distractor did not differ significantly, McNemar $\chi^{2}(1)=0.69, p=.405$. Repeating analysis of search RTs while excluding the 13 participants who correctly chose both frequent locations did not change the overall pattern observed of significant main effects of distractor value and location, but no significant interaction.

\section{Discussion}

In Experiment 2, high- and low-value distractors typically appeared in distinct locations. Under these conditions, we again found that search performance was impaired when the display contained a high-value distractor versus a low-value distractor, indicating an influence of reward learning on attentional priority during the search task. Experiment 2 also again found evidence of suppression resulting from statistical learning about frequent distractor locations: responses were faster when the distractor appeared in its most frequent location (frequentHigh for the high-value distractor; frequentLow for the low-value distractor) than when it appeared in one of the rare locations. Critically, however, we again found no evidence of an interaction between the effects of value and location, with Bayesian analysis providing strong support for the independence of these influences on performance in the search task. This is notable, since the design of Experiment 2 should have created a greater drive to suppress items appearing at the frequentHigh location, since suppressing high-reward distractors - which were most common at this frequentHigh location-was most beneficial for earning reward in this task. Nevertheless, no difference in suppression was observed at the frequentHigh and frequentLow locations. 
Consistent with prior research (Failing, Feldmann-Wüstefeld, et al., 2019) there was some evidence that the (reward-independent) suppression observed in Experiment 2 was feature-specific, in that responses were faster when the colour of the distractor on a given trial matched the colour of the distractor that most frequently appeared at that location, than when it mismatched; e.g., search performance was better when a blue distractor appeared at a location in which a blue distractor had appeared most frequently, versus when it appeared at a location in which an orange distractor had appeared most frequently.

That said, however, the suppression was not entirely feature-specific: responses on distractor-absent trials were slower when the target appeared in one of the frequent distractor locations than when it appeared in one of the rare distractor locations (as in Experiment 1). This latter finding suggests that, in addition to a feature-specific suppression of a particular colour at a particular frequent-distractor location, there was also a featuregeneral suppression that applied to any item presented at that location. One slightly discrepant result in this regard is that we did not see a significant difference in performance on distractor-present trials in the mismatch versus rare conditions: if there were some degree of general suppression at both frequent-distractor locations, then we should expect to see faster responding in the mismatch condition. The null result here may simply reflect noise in the data (mismatch trials were the least frequent and hence have greatest noise); we note that Figure 4 shows a (nonsignificant) numerical trend towards a mismatch-vs-rare difference, and Failing, Feldmann-Wüstefeld, et al. (2019) did find a significant mismatch-vsrare difference in their study which was considerably longer than ours (2,304 trials versus 896 in the current study, providing greater signal-to-noise ratio) but which did not manipulate reward. 
Failing, Feldmann-Wüstefeld, et al. (2019) took their data indicating both featurespecific and feature-general suppression—which mirror the current findings-as evidence that history-driven suppression operates on multiple levels of the visual processing hierarchy: at the level of feature maps (e.g., a saliency map for the colour blue, and a separate map for orange), and conspicuity maps (e.g., a colour map, which sums the individual feature maps for specific colours). Information from conspicuity maps for different dimensions (colour, shape, etc) is then fed forward to an overall priority map (Itti \& Koch, 2000, 2001). On this account, when a blue singleton appears in a location in which a blue distractor has often appeared in the past (match condition), it is subject to suppression both on the blue feature map and the colour conspicuity map. By contrast, when a blue singleton appears in a location in which an orange distractor has appeared in the past (mismatch condition), it receives suppression only at the level of the conspicuity map. Hence this approach correctly anticipates greater suppression in the match condition than the mismatch condition (for further detail, see Failing, Feldmann-Wüstefeld, et al., 2019; see also Gaspelin \& Luck, 2018, for related research).

\section{General Discussion}

A growing body of research indicates that prior experience can exert varied effects on attentional prioritisation of visual stimuli. Specifically, it has been shown learning about the value of rewards and punishments associated with a stimulus can increase the priority of that stimulus (for reviews, see Anderson, 2016; Le Pelley et al., 2016; Rusz et al., 2020; Watson, Pearson, Wiers, et al., 2019). A second stream of research has shown that statistical learning can result in attentional suppression of locations in which salient distractor stimuli are likely to appear (e.g., Failing, Feldmann-Wüstefeld, et al., 2019; Wang et al., 2019; Wang \& Theeuwes, 2018a, 2018b). 
The current study combined these two approaches, to investigate whether these two pathways - both based on selection history-operate independently, or whether they interact to determine the overall attentional priority of a given item in a given location. In both experiments, we found clear evidence of effects of both distractor value and distractor location on search performance. In line with previous work (Le Pelley et al., 2015; Watson, Pearson, Most, et al., 2019), participants were slower to respond to the search target when the display contained a distractor that signalled availability of high reward versus low reward. The implication is that the high-value distractor was more likely to receive attentional priority and hence interfere with search for the target, even though this pattern was counterproductive as the resulting slowing of responses had a negative impact on the overall reward that participants earned. Evidence of statistical learning was observed in that search performance was significantly better when the salient distractor appeared in a location in which distractors had frequently appeared in the past, versus a location in which distractors had seldom appeared. Moreover, participants were slower to respond to a target presented in a location in which distractors had frequently appeared, versus a location in which distractors had rarely appeared. These findings imply attentional suppression of the frequent-distractor location.

Critically, results of both experiments showed that the effects of distractor value and distractor location were additive, suggesting that these two factors exert independent effects on attentional priority. In Experiment 1, both high- and low-value distractors were most likely to occur in a single location. In this case, the independent effects of value and location indicate that a fixed, limited amount of suppression was applied to this frequent location, and this suppression applied equally to both types of distractor. Consequently, high-value distractors were more likely to 'break through' this suppression and capture attention, thus 
slowing search - an idea that is consistent with conclusions from previous research that has examined effects of reward in the context of other forms of inhibition (Pearson et al., 2020; Wang et al., 2014).

In Experiment 2, high- and low-value distractors were most likely to occur in different locations, creating a situation in which there should have been a greater drive to develop suppression at the frequent location of high-value distractors (since this would allow greater earnings on the most important, high-value trials). Nevertheless, once again the degree of suppression was uninfluenced by reward value: results indicated that a similar level of suppression applied at the frequent locations of high- and low-value distractors.

Our findings thus indicate that reward learning (about the value of distractors) and statistical learning (about the likely location of distractors) operate via separate pathways to influence attentional prioritisation. This conclusion is consistent with a view that sees attention as mediated by a common priority map that receives and integrates separate signals relating to physical salience and value (Awh et al., 2012; Belopolsky, 2015; Failing \& Theeuwes, 2018; Pearson et al., 2016; Theeuwes, 2018). In particular, our data suggest that suppression may be a product of learning based on the location of physically salient distractors, but is not affected by learning about the value associated with those distractors. This in turn implies that suppression develops at the level of feature/conspicuity maps relating to physical features (cf. Failing, Feldmann-Wüstefeld, et al., 2019), rather than in maps relating to value, or in response to salience in the integrated, common priority map (since in that case we would expect greater suppression at the location of the higher salience, high-value distractors).

In line with this suggestion, the results of Experiment 2 stand in notable contrast to those of a recent study by Failing and Theeuwes (2020), who investigated the effect of 
physical salience on suppression by varying the distinctiveness of a colour-singleton distractor relative to other items in the search display. Specifically, some trials of their task featured a high-salience distractor (a red shape that was very distinct from the other items in the display, which were all green), whereas other trials featured a low-salience distractor (a yellowish-green shape). Following the general approach of the current Experiment 2, the high-salience distractor was most likely to appear in one location, and the low-salience distractor was most likely to appear in a different location. Critically (and unlike the current study), Failing and Theeuwes found that this manipulation of physical salience generated significantly stronger suppression in the frequent location of the high-salience distractor than in the frequent location of the low-salience distractor. In summary, then, it seems that differences in physical salience of distractors can result in different levels of suppression arising from statistical learning, but differences in value (or incentive salience: Berridge $\&$ Robinson, 1998; Colaizzi et al., 2020; Pearson et al., 2020) do not. Again this conclusion is consistent with the idea that suppression develops at the level of salience maps relating to physical features, rather than maps encoding value.

In each of the current experiments, a minority of participants correctly identified the location in which distractors had most frequently appeared when asked to do so at the end of the task. Moreover, restricting analyses to only those participants who failed to identify the correct location(s) revealed a similar pattern of findings to overall analyses (main effects of value and location, with no interaction). Such findings have previously been taken to suggest that learning of statistical regularities is implicit in this task, and that the suppression generated by this learning is not mediated by explicit awareness. While our findings are in line with this view, we are wary of interpreting a participant's failure to select the correct location in a 'one-shot' awareness test at the end of a long experiment-when motivation is 
likely to be low-as evidence of a total lack of explicit knowledge (see Lovibond \& Shanks, 2002; Vadillo et al., 2016). It seems likely that such tests do not provide an exhaustive assessment of relevant conscious knowledge, and are not as sensitive in assessing that knowledge as is the test of effects on performance (based hundreds or thousands of trials in the search task). Consequently, failure on such awareness tests may not be as unambiguously diagnostic of implicit processes as previous work has implied. We remain agnostic on this issue, and note that the question of (un)awareness was not central to the current study.

We have characterised attention to the reward-signalling distractors in the current task as being counterproductive, since attending to distractors would slow responses to the target and hence reduce earnings: participants were (truthfully) informed that the best strategy was to ignore the coloured shapes altogether and simply respond as quickly as possible on every trial. Under these conditions, we take the observation of an attentional bias towards the high-value distractor to indicate a relatively automatic influence of value on attentional priority, wherein pairings of a distractor colour with high-value outcomes render that colour more salient and hence more likely to capture attention in future, regardless of the participant's current task-goals. However, we should consider the alternative possibility that participants monitored for the high-value distractor strategically, since this distractor had informational value (Gottlieb et al., 2014) -it signalled when a large reward was available-even though this was a poor strategy to use since it would result in lower earnings. This latter interpretation is unlikely, however. First, we have previously shown in a task using a similar reward manipulation that the pattern of greater attention to the highvalue distractor persists even when all rewards are removed, such that the distractors no longer have any informational value and hence there is no strategic reason to select them 
(Watson, Pearson, Most, et al., 2019; see also Le Pelley, Seabrooke, Kennedy, Pearson \& Most, 2017). Second, the data from the current task show that suppression was applied at the location of both the high- and low-value distractors, indicating a drive to prevent attention to these distractors, rather than to attend to them and make use of the value information that they provided. Hence we believe the effect of value on performance in the current task reflects a relatively automatic (and persistent) influence of value learning on attentional prioritisation, wherein signals of high-value outcomes become more likely to capture attention regardless of the participant's goals.

Our study has some limitations. First, the experiments were run online, as a consequence of COVID-19 preventing in-person testing. This reduced our degree of control over stimulus presentation (size, luminance etc) and testing conditions (distractions while participants were performing the task), which may have created additional noise in the data. Nevertheless, both experiments found clear evidence of effects of distractor value and location on performance, with large effect sizes, demonstrating that our implementation of the search task was indeed sensitive to detecting effects of the critical independent variables on performance. Second, in these experiments the presence of a high-value distractor (versus a low-value distractor) signalled that a correct response to the target would receive larger reward, and also that an incorrect response would lead to a larger loss of points. Under these conditions we cannot be sure whether the influence of value on attention observed here reflects an effect of the stimulus's relationship with reward, with punishment, or both. On the basis of prior research it seems likely that both have an effect: that the critical issue is the motivational significance of the outcome signalled by a stimulus, rather than the valence of that outcome (e.g., Le Pelley et al., 2019; Wang et al., 2013; Wentura et al., 2014; for review, see Watson, Pearson, Wiers, et al., 2019). For current purposes it was 
sufficient to show that performance in this task was influenced by 'value'—which incorporates both reward and punishment-but future research could study this issue more closely using a procedure in which correct responses are rewarded but errors are not punished (to investigate the effect of reward learning specifically), or in which errors are punished but correct responses are not rewarded (to investigate the effect of punishment). In summary, the current study investigated two aspects of selection history that have previously been shown to shape attentional priority: learning about the value of outcomes signalled by stimuli, and learning about the likely location in which salient distracting events will appear. Consistent with prior research, we found clear evidence of an effect of both of these factors on performance in a visual search task. Critically, our results indicate that these influences are additive, suggesting that learning about value and learning about location exert independent effects on attention to distractors: a finding which can be reconciled with an account in which attentional priority is mediated by a common priority map that receives and integrates signals relating to physical salience and reward. More generally, our findings sit within a growing body of research indicating that attention is not simply a function of stimulus features and top-down goals, but is instead critically influenced by our prior experiences - and extends this idea by showing that different effects of prior experiences may themselves be mediated by distinct cognitive pathways. 


\section{References}

Albertella, L., Le Pelley, M.E., Chamberlain, S.R., Westbrook, F., Fontenelle, L.F., Segrave, R., Lee, R., Pearson, D., \& Yücel, M. (2019). Reward-related attentional capture is associated with severity of addictive and obsessive-compulsive behaviors. Psychology of Addictive Behaviors, 33, 495-502. https://doi.org/10.1037/adb0000484

Albertella, L., Watson, P., Yücel, M., \& Le Pelley, M.E. (2019). Persistence of value-modulated attentional capture is associated with risky alcohol use. Addictive Behaviors Reports, 10, 100195. https://doi.org/10.1016/j.abrep.2019.100195

Anderson, B.A. (2016). The attention habit: How reward learning shapes attentional selection. Annals of the New York Academy of Sciences, 1369, 24-39. https://doi.org/10.1111/nyas.12957

Anderson, B.A., Laurent, P.A., \& Yantis, S. (2011). Learned value magnifies salience-based attentional capture. PLOS ONE, 6, 1-6. https://doi.org/10.1371/journal.pone.0027926

Awh, E., Belopolsky, A.V., \& Theeuwes, J. (2012). Top-down versus bottom-up attentional control: A failed theoretical dichotomy. Trends in Cognitive Sciences, 16, 437-443. https://doi.org/10.1016/j.tics.2012.06.010

Belopolsky, A.V. (2015). Common priority map for selection history, reward and emotion in the oculomotor system. Perception, 44, 920-933. https://doi.org/10.1177/0301006615596866

Berridge, K.C., \& Robinson, T.E. (1998). What is the role of dopamine in reward: hedonic impact, reward learning, or incentive salience? Brain Research Reviews, 28, 309-369. https://doi.org/10.1016/S0165-0173(98)00019-8

Colaizzi, J.M., Flagel, S.B., Joyner, M.A., Gearhardt, A.N., Stewart, J.L., \& Paulus, M.P. (2020). Mapping sign-tracking and goal-tracking onto human behaviors. Neuroscience $\&$ Biobehavioral Reviews, 111, 84-94. https://doi.org/10.1016/j.neubiorev.2020.01.018 de Leeuw, J.R. (2015). jsPsych: A JavaScript library for creating behavioral experiments in a web browser. Behavior Research Methods, 47, 1-12. https://doi.org/10.3758/s13428$\underline{014-0458-y}$

de Leeuw, J.R., \& Motz, B.A. (2016). Psychophysics in a Web browser? Comparing response times collected with JavaScript and Psychophysics Toolbox in a visual search task. Behavior Research Methods, 48, 1-12. https://doi.org/10.3758/s13428-015-0567-2 
Failing, M., Feldmann-Wüstefeld, T., Wang, B.C., Olivers, C., \& Theeuwes, J. (2019). Statistical regularities induce spatial as well as feature-specific suppression. Journal of Experimental Psychology: Human Perception and Performance, 45, 1291-1303. https://doi.org/10.1037/xhp0000701

Failing, M., \& Theeuwes, J. (2018). Selection history: How reward modulates selectivity of visual attention. Psychonomic Bulletin \& Review, 25, 514-538. https://doi.org/10.3758/s13423-017-1380-y

Failing, M., \& Theeuwes, J. (2020). More capture, more suppression: Distractor suppression due to statistical regularities is determined by the magnitude of attentional capture. Psychonomic Bulletin \& Review, 27, 86-95. https://doi.org/10.3758/s13423-019-01672-z Failing, M., Wang, B.C., \& Theeuwes, J. (2019). Spatial suppression due to statistical regularities is driven by distractor suppression not by target activation. Attention, Perception, \& Psychophysics, 81, 1405-1414. https://doi.org/10.3758/s13414-019$\underline{01704-9}$

Gao, Y., \& Theeuwes, J. (2020). Independent effects of statistical learning and top-down attention. Attention, Perception, \& Psychophysics. Advance online publication. https://doi.org/10.3758/s13414-020-02115-x

Gaspelin, N., \& Luck, S.J. (2018). Distinguishing among potential mechanisms of singleton suppression. Journal of Experimental Psychology: Human Perception and Performance, 44, 626-644. https://doi.org/10.1037/xhp0000484

Gottlieb, J., Hayhoe, M., Hikosaka, O., \& Rangel, A. (2014). Attention, reward, and information seeking. Journal of Neuroscience, 34, 15497-15504. https://doi.org/10.1523/JNEUROSCI.3270-14.2014

Itti, L., \& Koch, C. (2000). A saliency-based search mechanism for overt and covert shifts of visual attention. Vision Research, 40, 1489-1506. https://doi.org/10.1016/S00426989(99)00163-7

Itti, L., \& Koch, C. (2001). Computational modelling of visual attention. Nature Reviews Neuroscience, 2, 194-203. https://doi.org/10.1038/35058500

Jeffreys, H. (1961). Theory of probability. Oxford, UK: Oxford University Press.

Le Pelley, M.E., Mitchell, C.J., Beesley, T., George, D.N., \& Wills, A.J. (2016). Attention and associative learning in humans: An integrative review. Psychological Bulletin, 142, 1111 1140. https://doi.org/10.1037/bul0000064 
Le Pelley, M.E., Pearson, D., Griffiths, O., \& Beesley, T. (2015). When goals conflict with values: Counterproductive attentional and oculomotor capture by reward-related stimuli. Journal of Experimental Psychology: General, 144, 158-171. https://doi.org/10.1037/xge0000037

Le Pelley, M.E., Seabrooke, T., Kennedy, B.L., Pearson, D., \& Most, S.B. (2017). Miss it and miss out: Counterproductive nonspatial attentional capture by task-irrelevant, value-related stimuli. Attention, Perception, \& Psychophysics, 79, 1628-1642. https://doi.org/10.3758/s13414-017-1346-1

Le Pelley, M.E., Watson, P., Pearson, D., Abeywickrama, R.S., \& Most, S.B. (2019). Winners and losers: Reward and punishment produce biases in temporal selection. Journal of Experimental Psychology: Learning, Memory, \& Cognition, 45, 822-833. https://doi.org/10.1037/xlm0000612

Lovibond, P.F., \& Shanks, D.R. (2002). The role of awareness in Pavlovian conditioning: Empirical evidence and theoretical implications. Journal of Experimental Psychology: Animal Behavior Processes, 28, 3-26. https://doi.org/10.1037/0097-7403.28.1.3

Morey, R.D. (2008). Confidence intervals from normalized data: A correction to Cousineau (2005). Tutorial in Quantitative Methods for Psychology, 4, 61-64. https://doi.org/10.20982/tamp.04.2.p061

Pearson, D., Donkin, C., Tran, S.C., Most, S.B., \& Le Pelley, M.E. (2015). Cognitive control and counterproductive oculomotor capture by reward-related stimuli. Visual Cognition, 23, 41-66. https://doi.org/10.1080/13506285.2014.994252

Pearson, D., Osborn, R., Whitford, T.J., Failing, M., Theeuwes, J., \& Le Pelley, M.E. (2016). Value-modulated oculomotor capture by task-irrelevant stimuli is a consequence of early competition on the saccade map. Attention, Perception, \& Psychophysics, 78, 2226-2240. https://doi.org/10.3758/s13414-016-1135-2

Pearson, D., Watson, P., Cheng, P., \& Le Pelley, M.E. (2020). Overt attentional capture by reward-related stimuli overcomes inhibitory suppression. Journal of Experimental Psychology: Human Perception and Performance, 46, 489-501.

\section{https://doi.org/10.1037/xhp0000728}

Rusz, D., Le Pelley, M.E., Kompier, M.A.J., Mait, L., \& Bijleveld, E. (2020). Reward-driven distraction: A meta-analysis. Psychological Bulletin. Advance online publication. https://doi.org/10.1037/bul0000296 
The jamovi project. (2020). jamovi (Version 1.2) [Computer software]. https://www.jamovi.org Theeuwes, J. (1992). Perceptual selectivity for color and form. Perception \& Psychophysics, 51 , 599-606. https://doi.org/10.3758/Bf03211656

Theeuwes, J. (2018). Visual selection: Usually fast and automatic; seldom slow and volitional. J Cogn, 1, 29. https://doi.org/10.5334/joc. 13

Vadillo, M.A., Konstantinidis, E., \& Shanks, D.R. (2016). Underpowered samples, false negatives, and unconscious learning. Psychonomic Bulletin \& Review, 23, 87-102. https://doi.org/10.3758/s13423-015-0892-6

Wang, B., Samara, I., \& Theeuwes, J. (2019). Statistical regularities bias overt attention. Attention, Perception, \& Psychophysics, 81, 1813-1821. https://doi.org/10.3758/s13414$\underline{019-01708-5}$

Wang, B.C., \& Theeuwes, J. (2018a). Statistical regularities modulate attentional capture. Journal of Experimental Psychology: Human Perception and Performance, 44, 13-17. https://doi.org/10.1037/xhp0000472

Wang, B.C., \& Theeuwes, J. (2018b). Statistical regularities modulate attentional capture independent of search strategy. Attention, Perception, \& Psychophysics, 80, 1763-1774. https://doi.org/10.3758/s13414-018-1562-3

Wang, L., Duan, Y., Theeuwes, J., \& Zhou, X. (2014). Reward breaks through the inhibitory region around attentional focus. Journal of Vision, 14, 1-7. https://doi.org/10.1167/14.12.2

Wang, L., Yu, H., \& Zhou, X. (2013). Interaction between value and perceptual salience in value-driven attentional capture. Journal of Vision, 13, 1-13. https://doi.org/10.1167/13.3.5

Watson, P., Pearson, D., Chow, M., Theeuwes, J., Wiers, R.W., Most, S.B., \& Le Pelley, M.E. (2019). Capture and control: Working memory modulates attentional capture by reward-related stimuli. Psychological Science, 30, 1174-1185. https://doi.org/10.1177/0956797619855964

Watson, P., Pearson, D., Most, S.B., Theeuwes, J., Wiers, R.W., \& Le Pelley, M.E. (2019). Attentional capture by Pavlovian reward-signalling distractors in visual search persists when rewards are removed. PLOS ONE, 14, e0226284.

https://doi.org/10.1371/journal.pone.0226284 
Watson, P., Pearson, D., Wiers, R.W., \& Le Pelley, M.E. (2019). Prioritizing pleasure and pain: Attentional capture by reward-related and punishment-related stimuli. Current Opinion in Behavioral Sciences, 26, 107-113. https://doi.org/10.1016/j.cobeha.2018.12.002

Wentura, D., Müller, P., \& Rothermund, K. (2014). Attentional capture by evaluative stimuli: Gain- and loss-connoting colors boost the additional-singleton effect. Psychonomic Bulletin \& Review, 21, 701-707. https://doi.org/10.3758/s13423-013-0531-z

Yantis, S. (2000). Goal-directed and stimulus-driven determinants of attentional control. In S. Monsell \& J. Driver (Eds.), Attention and Performance XVIII (pp. 73-103). Cambridge, MA: MIT Press. 